Synthesis and Properties of a Low-Bandgap Liquid Crystalline $\pi$-Conjugated Polymer Hiromasa Goto,* Aohan Wang, Kohsuke Kawabata, Fan Yang

Journal of Materials Science, 48(21), 7523-7532, (2013)

Synthesis and Properties of a Low-Bandgap Liquid Crystalline m-Conjugated Polymer

Hiromasa Goto, ${ }^{*}$ Aohan Wang, Kohsuke Kawabata, Fan Yang

Division of Materials Science, Faculty of Pure and Applied Sciences, University of Tsukuba, Tsukuba, Ibaraki 305-8573, Japan.

*Correspondence to H. Goto, email:gotoh@ims.tsukuba.ac.jp, fax: +81-298-53-4490

Highlights

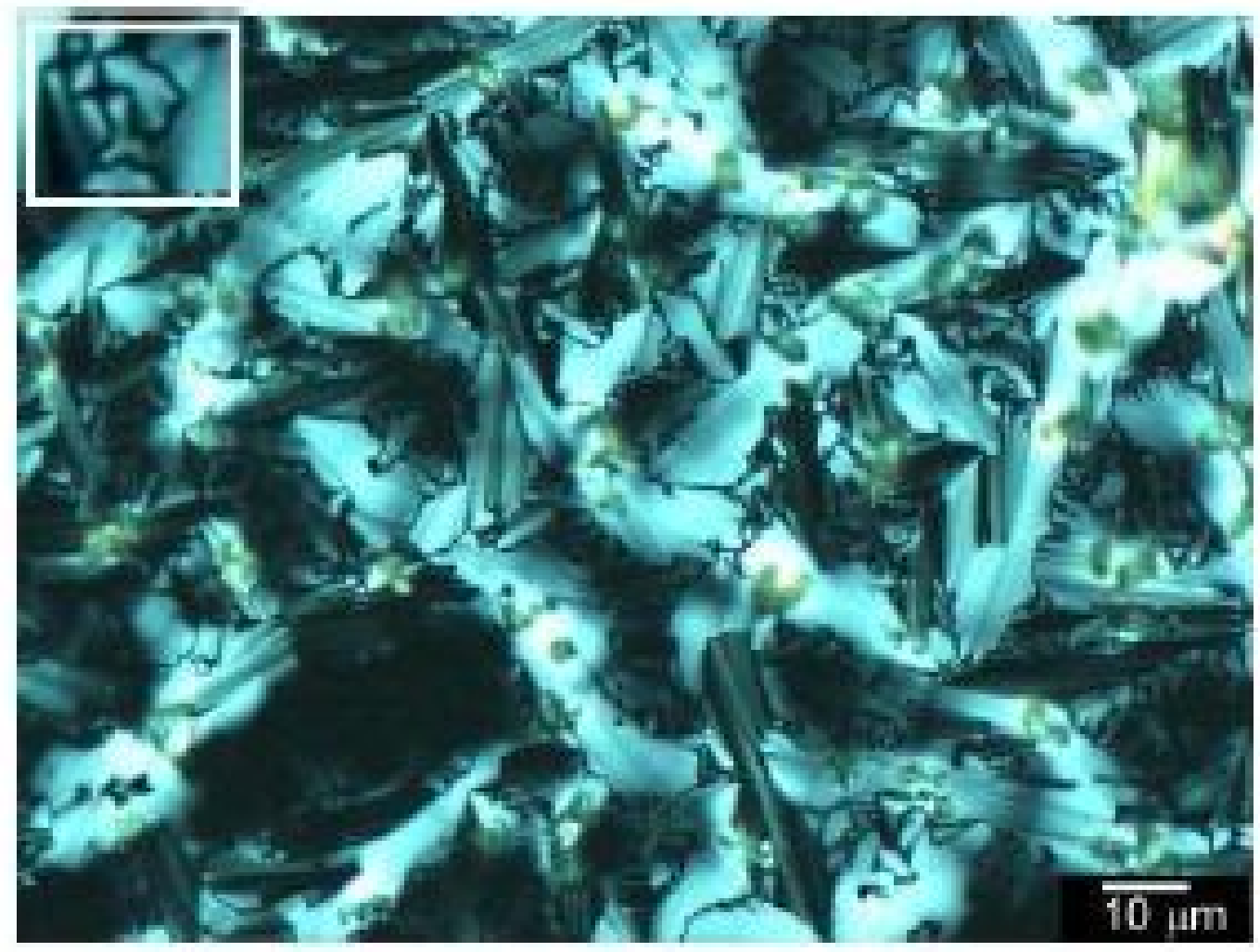

-An isothianaphthene-based liquid crystal conjugated polymer was successfully synthesized.

- The polymer shows both thermotropic and lyotropic liquid crystallinities.

- Preparation of an opto-electroactive liquid crystalline conjugated polymer has been achieved.

-New approach to the optically functional electro-plastics. 


\title{
Synthesis and Properties of a Low-Bandgap Liquid Crystalline $\pi$-Conjugated Polymer
}

\author{
Hiromasa Goto,* Aohan Wang, Kohsuke Kawabata, Fan Yang \\ Division of Materials Science, Faculty of Pure and Applied Sciences, University of \\ Tsukuba, Tsukuba, Ibaraki 305-8573, Japan. \\ *Correspondence to H. Goto, email:gotoh@ims.tsukuba.ac.jp, fax: +81-298-53-4490
}

Keywords: electrochromism; electroactive, CIE; liquid crystal; low-bandgap.

Keywords: electrochromism; electroactive, CIE; liquid crystal; low-bandgap.

\begin{abstract}
The synthesis and properties of an isothianaphthene-based liquid crystal (LC) $\pi$-conjugated polymer are reported. The polymer showed amphotropic LC character having both thermotropic and lyotropic liquid crystallinities with macroscopic LC fractal structure. The optical bandgap is $2.0 \mathrm{eV}$ accompanied by good electroactivity.
\end{abstract}

\section{Introduction}

Low-bandgap conjugated polymers have received much attention for applications in solar cells, batteries and display devices [1-12]. There are several strategies for preparation of the low-bandgap polymers. Introduction of isothianaphthene (ITN) units or methine in the main chain results in a low-bandgap polymer. However, the rigid skeletons derived from a highly developed conjugated system result in low processability [13].

The orientation of $\pi$-conjugated polymers is important for enhancement of their electro-optical functionality. For example, light emission from anisotropic structures shows polarized emission. Polarized light detection can be useful for sensors. However, improved processability is required for practical applications.

Liquid crystals (LCs) possess both orientational order and fluidity. The orientation of liquid crystalline material through external mechanical force, magnetic field, electric field, and polarized light provides anisotropy. The introduction of LC groups into $\pi$-conjugated polymers improves processability and orientational characteristics. However, the introduction causes expansion of the bandgap, accompanied by an 
Synthesis and Properties of a Low-Bandgap Liquid Crystalline $\pi$-Conjugated Polymer

Hiromasa Goto, ${ }^{*}$ Aohan Wang, Kohsuke Kawabata, Fan Yang

Journal of Materials Science, 48(21), 7523-7532, (2013)

increase of ionization potentials and decrease of electron affinity.

In this research, we considered a combination of isothianaphthene moiety and LC for the preparation of low-bandgap conjugated polymers. A pyrimidine-type LC group was employed in this study because of its highly stable liquid crystallinity. Nitrogen atoms at the pyrimidine mesogenic core aid intermolecular interactions to form LC molecular aggregation. The mesogens (LC groups) are introduced on both sides of the benzene ring of the LC unit. This structure has good regularity because of the lack of head-to-tail structure. The introduction of the ITN unit in the main chain can compensate expansion of the bandgap stemming from the introduction of the LC groups.

The polymer synthesized by Migita-Kosugi-Stille type polycondensation showed both thermotropic and lyotropic liquid crystallinity, which is referred to as amphotropic LC. The LC textures, LC emission (photoluminescence), and macroscopic fractal LC form of the polymer are discussed.

\section{Monomer synthesis}

Compound 1 was prepared by the Mitsunobu reaction [14]. Diethyl azodicarboxylate (DEAD) and triphenyl phosphine (TPP) were reacted before the coupling reaction between the alcohols. A mixture of DEAD in toluene (DEAD/toluene solution: 2.2 $\mathrm{mol} / \mathrm{L})(4.35 \mathrm{~g}, 25 \mathrm{mmol})$, and TPP $(2.62 \mathrm{~g}, 10 \mathrm{mmol})$ was stirred in $10 \mathrm{~mL}$ of tetrahydrofuran (THF) for $30 \mathrm{~min}$. Then 2,5-dibromobenzene-1,4-diol (1.4 g, $5 \mathrm{mmol}$ ) and 10-bromo-decan-1-ol (2.5 g, $10 \mathrm{mmol})$ were added to the red-colored solution and stirred for $12 \mathrm{~h}$ at room temperature. The evaporation of the solvent and purification by column chromatography ( silica gel, chloroform) yielded $0.564 \mathrm{~g}$ of compound 1 ( $\mathrm{Y}=$ $16 \%$, white powder).

The pyrimidine-based LC precursor was coupled with compound 1 by the Williamson etherification reaction to yield mono1. A mixture of compound $1(0.25 \mathrm{~g}$, $0.35 \mathrm{mmol})$, 4-(5-heptyl-pyrimidin-2-yl)-phenol (0.2 g, $0.74 \mathrm{mmol}), \mathrm{K}_{2} \mathrm{CO}_{3}(0.1 \mathrm{~g}, 0.72$ $\mathrm{mmol})$ and $\mathrm{KI}(0.12 \mathrm{~g}, 0.72 \mathrm{mmol})$ in $3 \mathrm{~mL}$ of 2-butanone was refluxed at $80{ }^{\circ} \mathrm{C}$ for $24 \mathrm{~h}$, followed by the solvent evaporation. Recrystallization from acetone yielded $0.25 \mathrm{~g}$ of mono1 ( $\mathrm{Y}=64.1 \%$, white powder). The synthetic routes are shown in Scheme 1 .

Trimethysilyl-substituted isothianaphthene at $\alpha, \alpha^{\prime}$-positions (mono2) was prepared by the previously reported method $[15,16]$. Lithiation of the unsubstituted isothianaphthene at $\alpha, \alpha^{\prime}$-positions was carried out in THF solution at dry ice temperature. Trimethyltin chloride was reacted with lithium at both $\beta$-positions of the isothianaphthene unit to obtain a di-substituted isothianaphthene with trimethyltin 
(mono2). Figure 1 shows ${ }^{1} \mathrm{H}$ NMR spectra of mono1 and mono2 in $\mathrm{CDCl}_{3}$ solution. Protons at carbons adjacent to the ether oxygen appear at around $4 \mathrm{ppm}$ from tetramethylsilane (TMS) (Figure 1(a)). The signals of aromatic protons are observable at the low magnetic regions. Methyl groups of mono2 are observed at $0.5 \mathrm{ppm}$ (Figure 1(b)). Aromatic protons of mono2 can be seen at 7.07 and $7.67 \mathrm{ppm}$. These results indicate that the monomers were successfully synthesized.

Mono1: ${ }^{1} \mathrm{H}$ NMR (400 MHz, $\delta$ from TMS (ppm), $\left.\mathrm{CDCl}_{3}\right): \delta 0.89$ (t, $6 \mathrm{H},-\left(\mathrm{CH}_{2}\right)_{8}-\mathrm{CH}_{3}$, $J=14 \mathrm{~Hz}), 1.2-1.8\left(\mathrm{~m}, 26 \mathrm{H},-\left(\mathrm{CH}_{2}\right)_{5}-\mathrm{CH}_{3},-\left(\mathrm{CH}_{2}\right)_{8}-\mathrm{CH}_{3}\right), 2.60\left(\mathrm{t}, 4 \mathrm{H},-\mathrm{CH}_{2}-\left(\mathrm{CH}_{2}\right)_{5}-\right)$, 3.93-4.05 (m, 8H, -O-CH $\left.2^{-}\right), 6.98$ (d, 2H, 2,5- $H$ (dibromobenzene), $\left.J=8.4 \mathrm{~Hz}\right), 7.08$ (s, $4 \mathrm{H}, 2,5 H$ (benzene)), 8.45 (d, 4H, 3,5H (benzene), $J=8.8 \mathrm{~Hz}), 8.57$ (s, 8H, $3,5 H$ (pyrimidine)).

Mono2: ${ }^{1} \mathrm{H}$ NMR (400 MHz, $\delta$ from TMS (ppm), $\left.\mathrm{CDCl}_{3}\right): \delta 0.49\left(\mathrm{~s}, 18 \mathrm{H}, \mathrm{CH}_{3}\right), 7.07$ $(\mathrm{dd}, 2 \mathrm{H}, 5,6-H, J=3.0,6.6 \mathrm{~Hz}), 7.67(\mathrm{dd}, 2 \mathrm{H}, 4,7-H, J=2.8,6.8 \mathrm{~Hz}) .{ }^{13} \mathrm{C} \mathrm{NMR}(400$ $\mathrm{MHz}, \delta$ from TMS (ppm), $\left.\mathrm{CDCl}_{3}\right): \delta 8.0,122.6,123.9,137.0,146.7$.
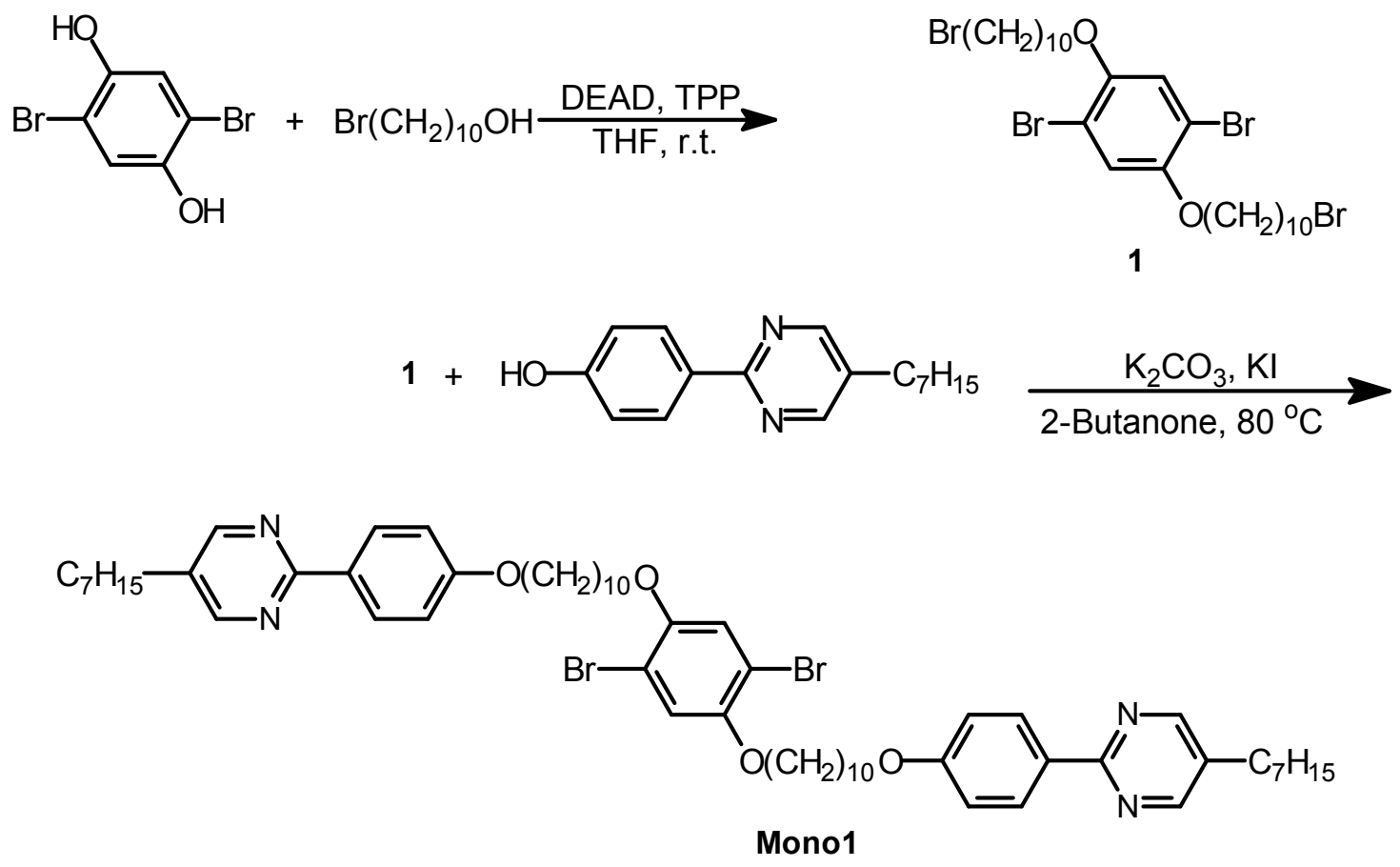

Scheme 1. Synthesis of pyrimidine monomer. DEAD = diethylazodicarboxylate, $\mathrm{TPP}=$ triphenylphosphine, THF $=$ tetrahydrofuran. 
Synthesis and Properties of a Low-Bandgap Liquid Crystalline $\pi$-Conjugated Polymer Hiromasa Goto, * Aohan Wang, Kohsuke Kawabata, Fan Yang

Journal of Materials Science, 48(21), 7523-7532, (2013)

(a)

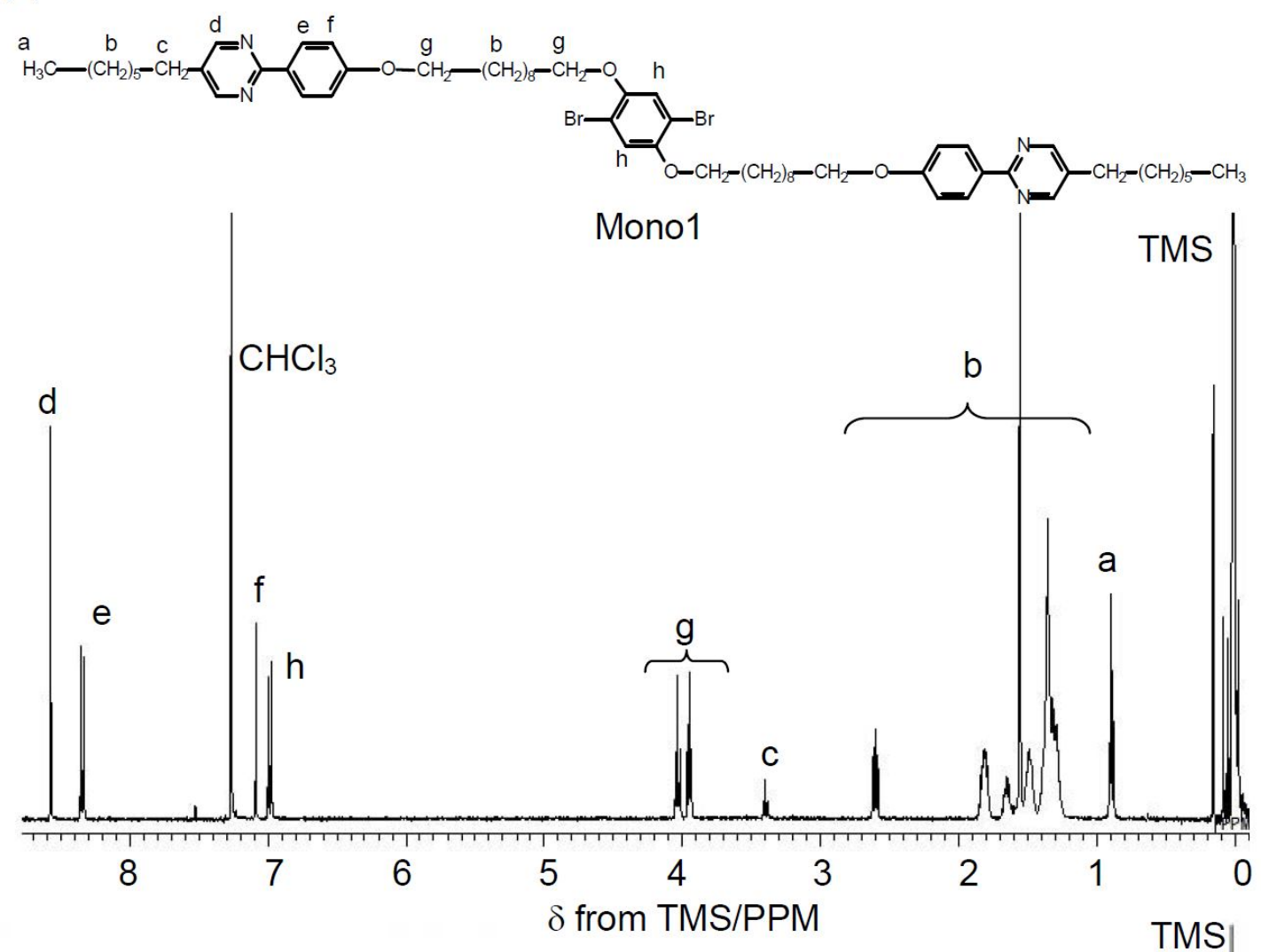

(b)

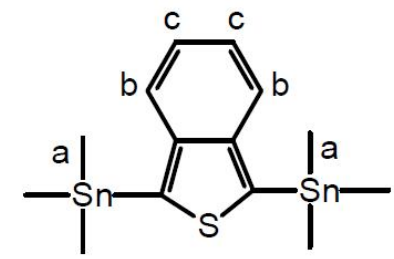

Mono2

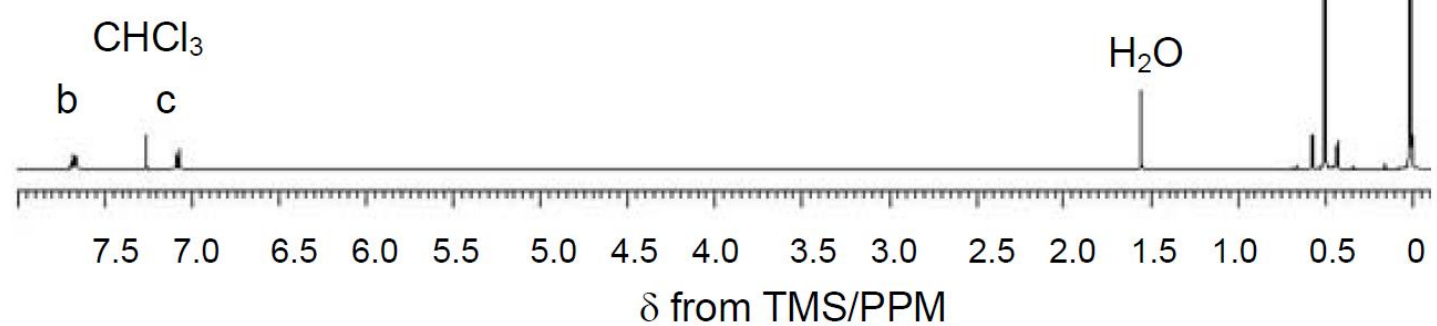

Fig. 1. ${ }^{1} \mathrm{H}$ NMR of monol (a) and mono2 (b) in $\mathrm{CDCl}_{3}$. TMS = tetramethyl silane. 
Synthesis and Properties of a Low-Bandgap Liquid Crystalline $\pi$-Conjugated Polymer Hiromasa Goto,* Aohan Wang, Kohsuke Kawabata, Fan Yang Journal of Materials Science, 48(21), 7523-7532, (2013)

\section{Copolymerization}

Copolymerization between mono1 and mono2 having LC groups was performed by Migita-Kosugi-Stille coupling reaction with the aid of $\operatorname{Pd}(0)$ catalyst (Scheme 2).

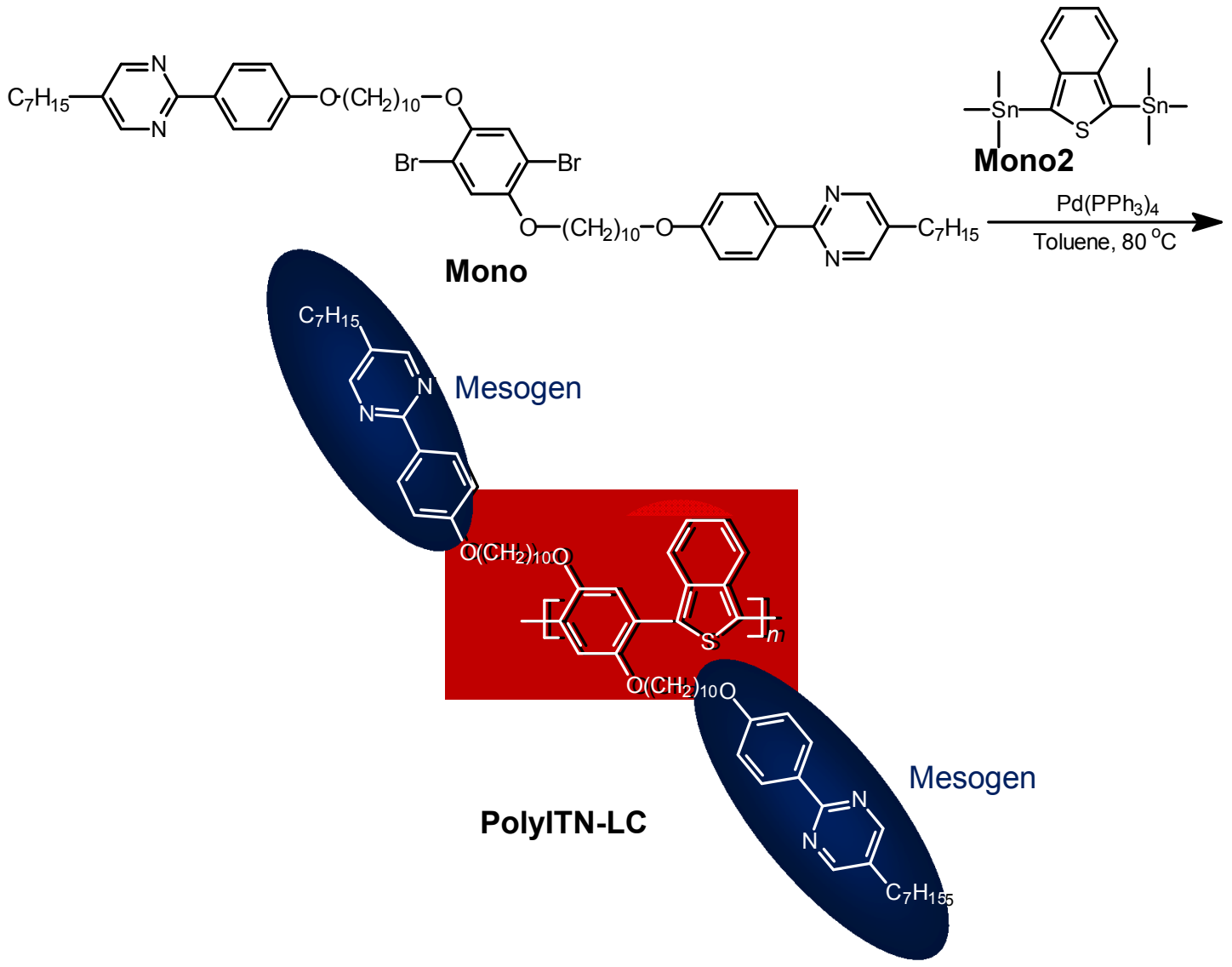

Scheme 2. Copolymerization and molecular structure of resultant polyITN-LC. $\operatorname{Pd}\left(\mathrm{PPh}_{3}\right)_{4}=$ tetrakis(triphenylphosphine)palladium $(0)$.

A mixture of mono1 (127 mg, $0.117 \mathrm{mmol})$ and mono $2(55 \mathrm{mg}, 0.124 \mathrm{mmol})$ in toluene $(0.6 \mathrm{~mL})$ was stirred. Then $37 \mathrm{mg}(0.032 \mathrm{mmol})$ of tetrakis (triphenylphosphine) palladium(0) $\left[\mathrm{Pd}\left(\mathrm{PPh}_{3}\right)_{4}\right]$ was added to the solution and it was refluxed at $80{ }^{\circ} \mathrm{C}$. The initially transparent polymerization solution turned dark red as the reaction progressed. After $12 \mathrm{~h}$, the reaction mixture was washed with a large amount of methanol, and the resultant precipitate was filtered and dried in vacuum to afford a dark-red solid as the desired product $\left(82 \mathrm{mg}, \mathrm{Y}=66 \%\right.$ ). The weight-average molecular weight $\left(M_{\mathrm{w}}\right)$ was 5650 , the number-average molecular weight $\left(M_{\mathrm{w}}\right)$ was 5200 , and the dispersity $\left(M_{\mathrm{n}} / M_{\mathrm{w}}\right)$ was 1.1 vs. the polystyrene standard, evaluated by the gel permeation chromatography 
(GPC). The polymer thus prepared is abbreviated as polyITN-LC. ${ }^{1} \mathrm{H}$ NMR measurement confirms chemical structure polyITN-LC (Figure 2).
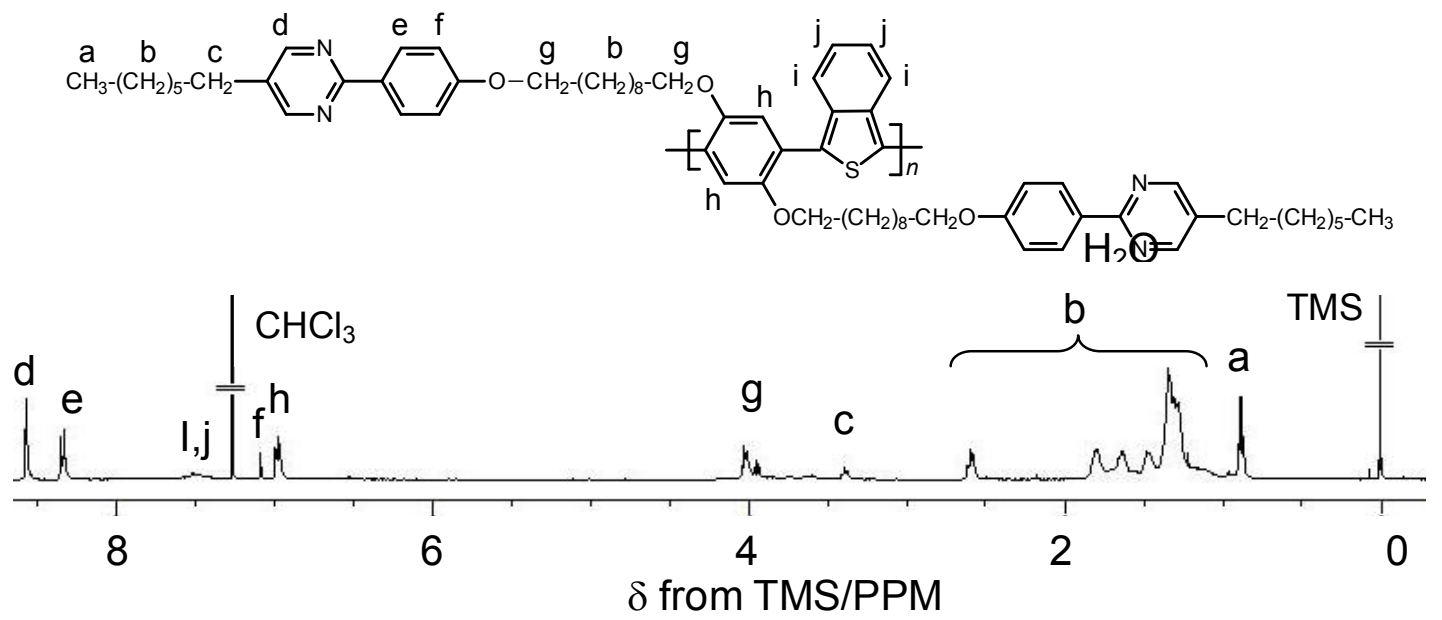

Fig. 2. ${ }^{1} \mathrm{H}$ NMR of the polymer in $\mathrm{CDCl}_{3}$. TMS = tetramethyl silane.

Additionally, synthesis of unsubstituted poly(phenylene-co-isothianaphthene) was carried out (Scheme 3). Firstly, a solution of mono1 $(15.7 \mathrm{mg}, 0.033 \mathrm{mmol}$ ) and $p$-diiodobenzene (18.9 mg, $0.057 \mathrm{mmol})$ in $N, N$-dimethylformamide (DMF, $1.0 \mathrm{~mL}$ ) was stirred. Then, $37 \mathrm{mg}(0.011 \mathrm{mmol})$ of $\left[\mathrm{Pd}\left(\mathrm{PPh}_{3}\right)_{4}\right]$ was added to the solution then refluxed at $85{ }^{\circ} \mathrm{C}$. The initially transparent polymerization solution turned dark red as the reaction progressed. After $72 \mathrm{~h}$, the reaction mixture was washed with a large amount of methanol, and the resultant precipitate was filtered and dried in vacuum to afford a dark-red solid as the desired product (abbreviated as polyITN-P, $\mathrm{P}=$ phenylene). $\mathrm{Y}=74 \%(5.8 \mathrm{mg})$. The dark red polymer thus obtained is insoluble and infusible.

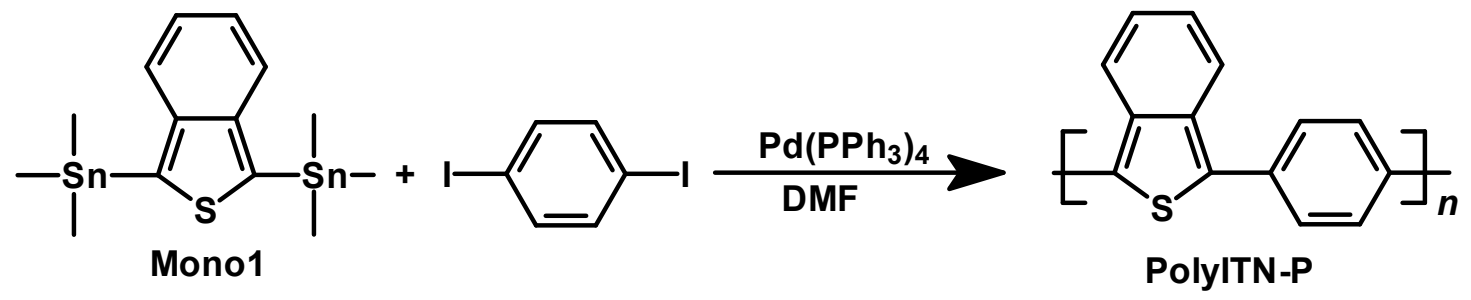

Scheme 3. Copolymerization and molecular structure of resultant polyITN-P. DMF $N$, $N$-dimethylformamide

\section{Results and discussion}

\subsection{Infrared absorption spectra}

Infrared (IR) absorption spectra of the monomer and the polymer are shown in Figure 3. 
Mono 1 shows absorption bands at $3000-2820 \mathrm{~cm}^{-1}$ attributable to $v_{\mathrm{CH} 2}$ and $v_{\mathrm{CH} 3}$ of the methylene groups. An intense absorption band corresponding to $v_{\mathrm{C}-\mathrm{O}-\mathrm{C}}$ as an ether stretching between benzene and flexible alkyl spacer of the LC unit was observed at $1245 \mathrm{~cm}^{-1}$ for monol and polyITN-LC. The absorption bands at $1613 \mathrm{~cm}^{-1}$ and 1585 $\mathrm{cm}^{-1}$ are due to $\mathrm{C}=\mathrm{C}$ stretching of the benzene ring. The absorption at $1430 \mathrm{~cm}^{-1}$ may be due to $\mathrm{CH}$ bend and $\mathrm{CH}$ stretching in the benzene ring of the ITN unit $[17,18]$. Insoluble and infusible polyITN-P shows broad absorptions. Absorption bands at 1662 $\mathrm{cm}^{-1}$ and $1558 \mathrm{~cm}^{-1}$ due to $\mathrm{C}=\mathrm{C}$ stretching of the benzene ring are confirmed.

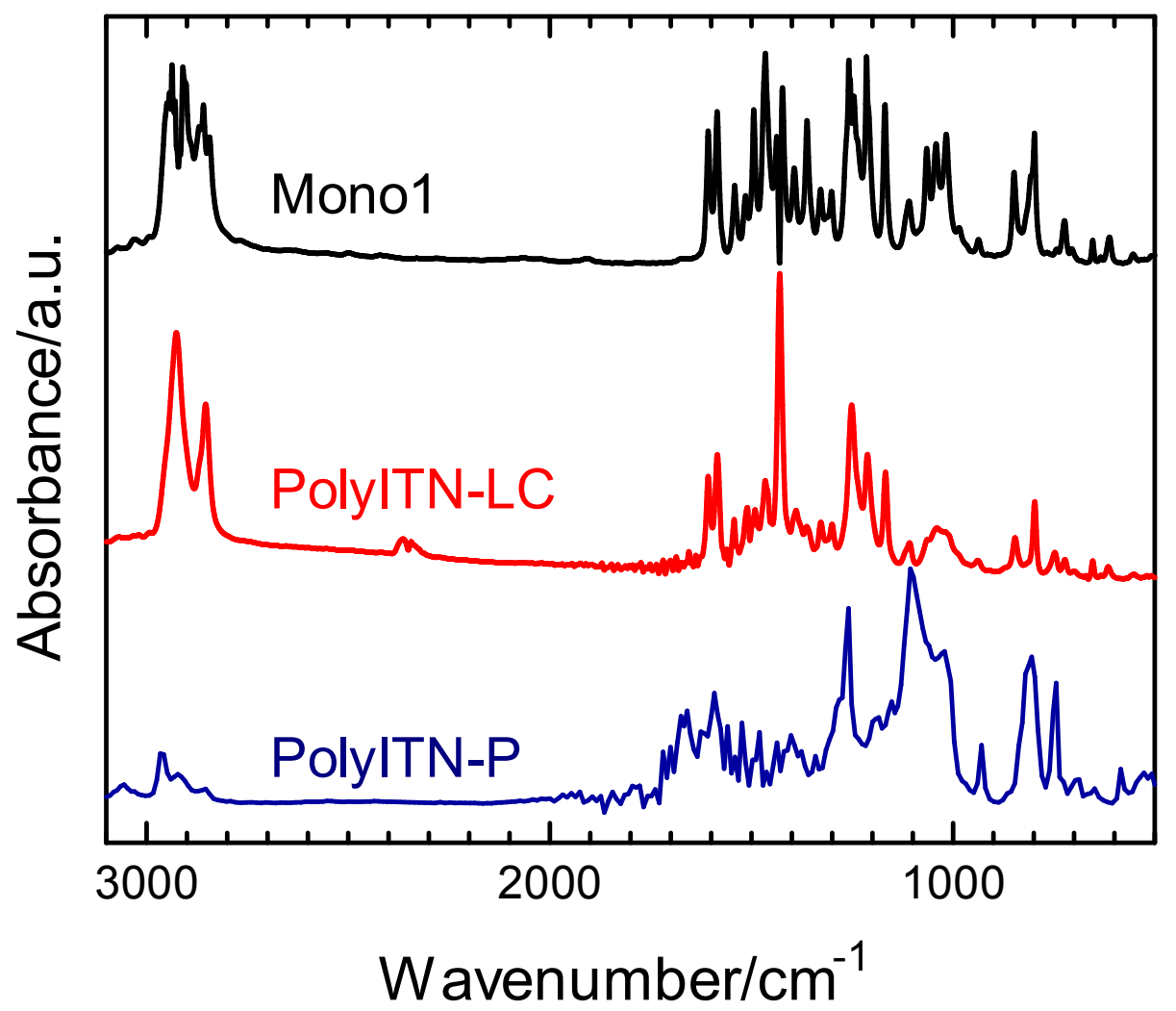

Fig. 3. IR spectra of mono1, polyITN-LC, and polyITN-P.

\subsection{Liquid crystallinity}

Liquid crystallinity of the compounds was examined by visual inspection with polarizing optical microscopy (POM) and differential scanning calorimetry (DSC). Figure 4(a) shows the POM image of mono1. The optical texture can be ascribed to smectic $\mathrm{C}$ phase $(\mathrm{SmC})$ possessing a tilted layer structure because fingerprint lines are observable on the fan-shaped structure. A possible molecular aggregation form of the mono1 in the SmC is shown in Figure 4(b). The entire molecular form consists of two side chains, while the phenylene moiety in the center (mono1) can behave as a mesogen to exhibit a SmC structure. 
Synthesis and Properties of a Low-Bandgap Liquid Crystalline $\pi$-Conjugated Polymer Hiromasa Goto, * Aohan Wang, Kohsuke Kawabata, Fan Yang

Journal of Materials Science, 48(21), 7523-7532, (2013)
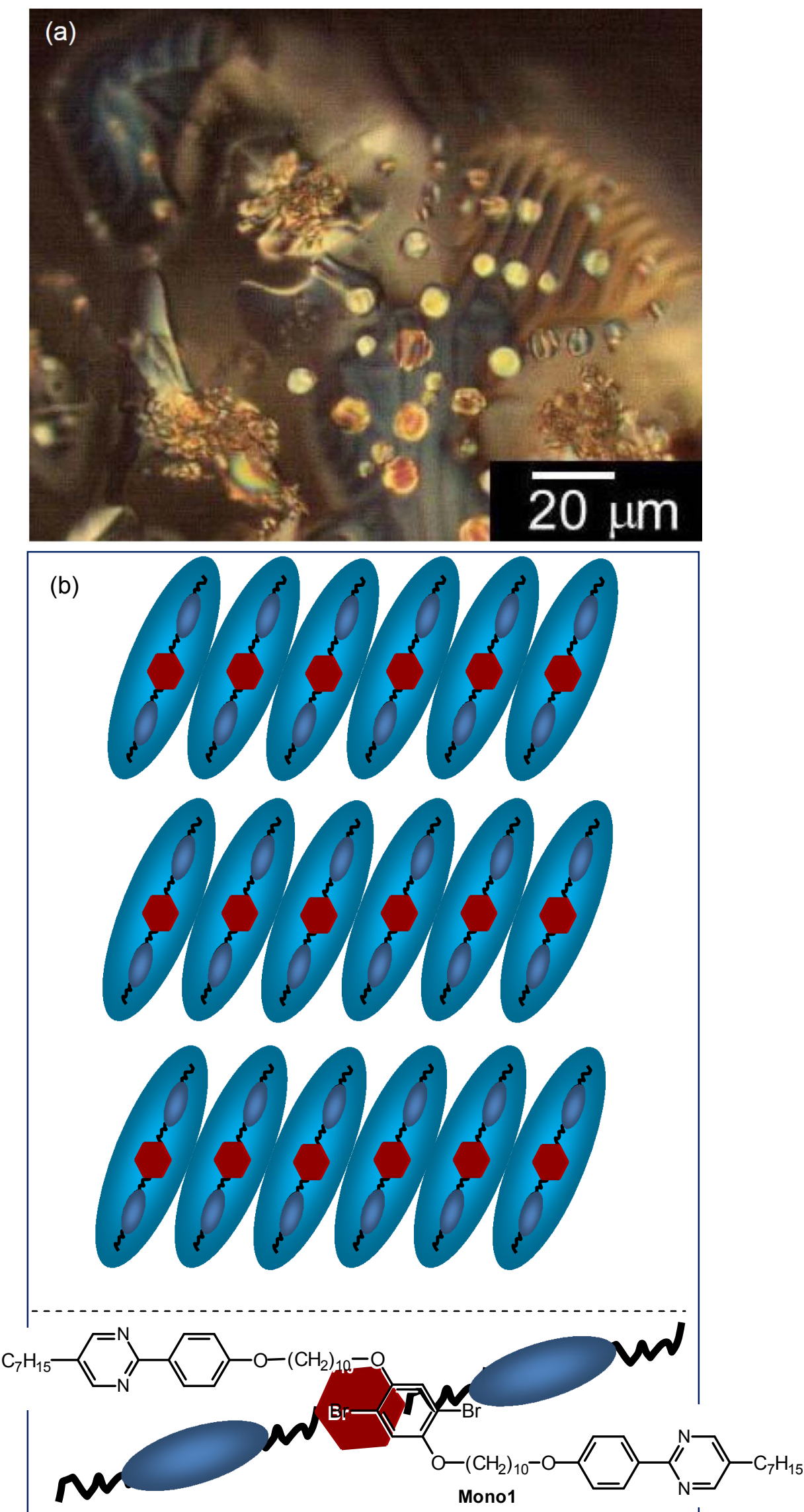
Synthesis and Properties of a Low-Bandgap Liquid Crystalline $\pi$-Conjugated Polymer Hiromasa Goto, ${ }^{*}$ Aohan Wang, Kohsuke Kawabata, Fan Yang

Journal of Materials Science, 48(21), 7523-7532, (2013)

Fig. 4. (a) Polarizing optical microscopy image of mono1. (b) Molecular aggregation form in SmC.

Figure 5 shows optical textures of the corresponding polymer (abbreviated as polyITN-LC) during heating (Figure 5(a)-(c)) and cooling processes (Figure 5(d)-(f)). The polymer clearly shows a fan-shaped texture of SmA. The dotted circle in Figure 5(a) indicates a diamond structure of the polymer, which is the evidence supporting formation of SmA. Schematic illustration of the diamond structure is shown in Figure $5(\mathrm{~g})$. The four fan-shaped domains produce a diamond structure in the texture. The absence of striped structure of the polymer indicates the absence of a tilted LC structure, such as SmC. The red color of the thick sample in the present observation comes from the original color of a $\pi$-conjugated main chain. The rigid main chain affords arrangement of the side chains in the perpendicular direction relative to the main chain to show SmA, as indicated in Figure 6. The main chain controls the arrangement of the side chains in the liquid crystal (LC) form that may be termed a "polymer effect in LC". Upon heating, the multi-domain texture gradually changed into isotropic phase (the black region in the images). The cooling process from isotropic allows formation of the
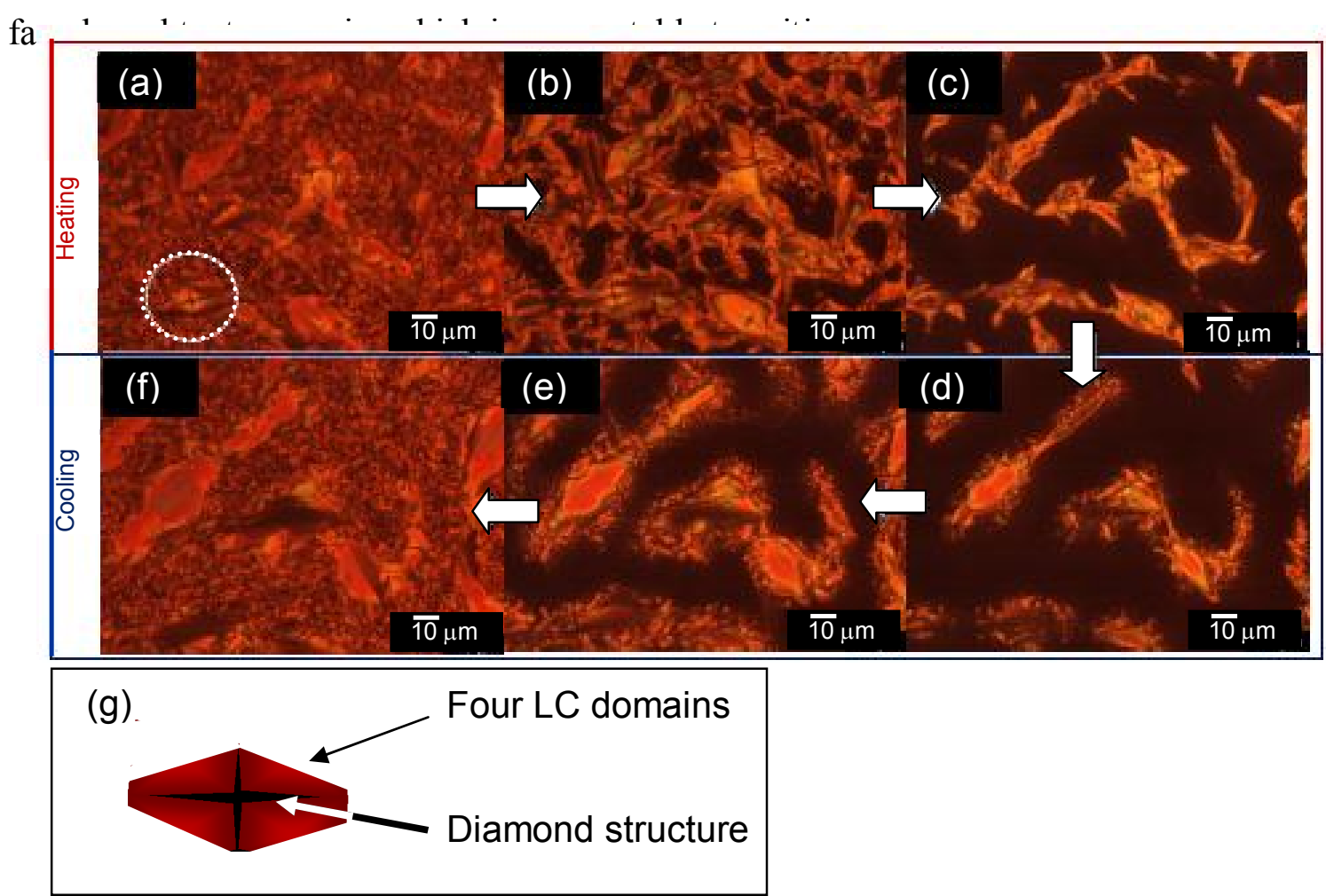

Fig. 5. Changes in polarizing optical microscopy images of polyITN-LC on cooling and heating. Heating process: (a) $40^{\circ} \mathrm{C}$, (b) $69^{\circ} \mathrm{C}$, (c) $74^{\circ} \mathrm{C}$. Cooling process: (d) $70^{\circ} \mathrm{C}$, (e) $66^{\circ} \mathrm{C}$, (f) $48^{\circ} \mathrm{C}$. Dotted circle shows diamond structure as evidence of SmA. (g) Formation of diamond structure. 


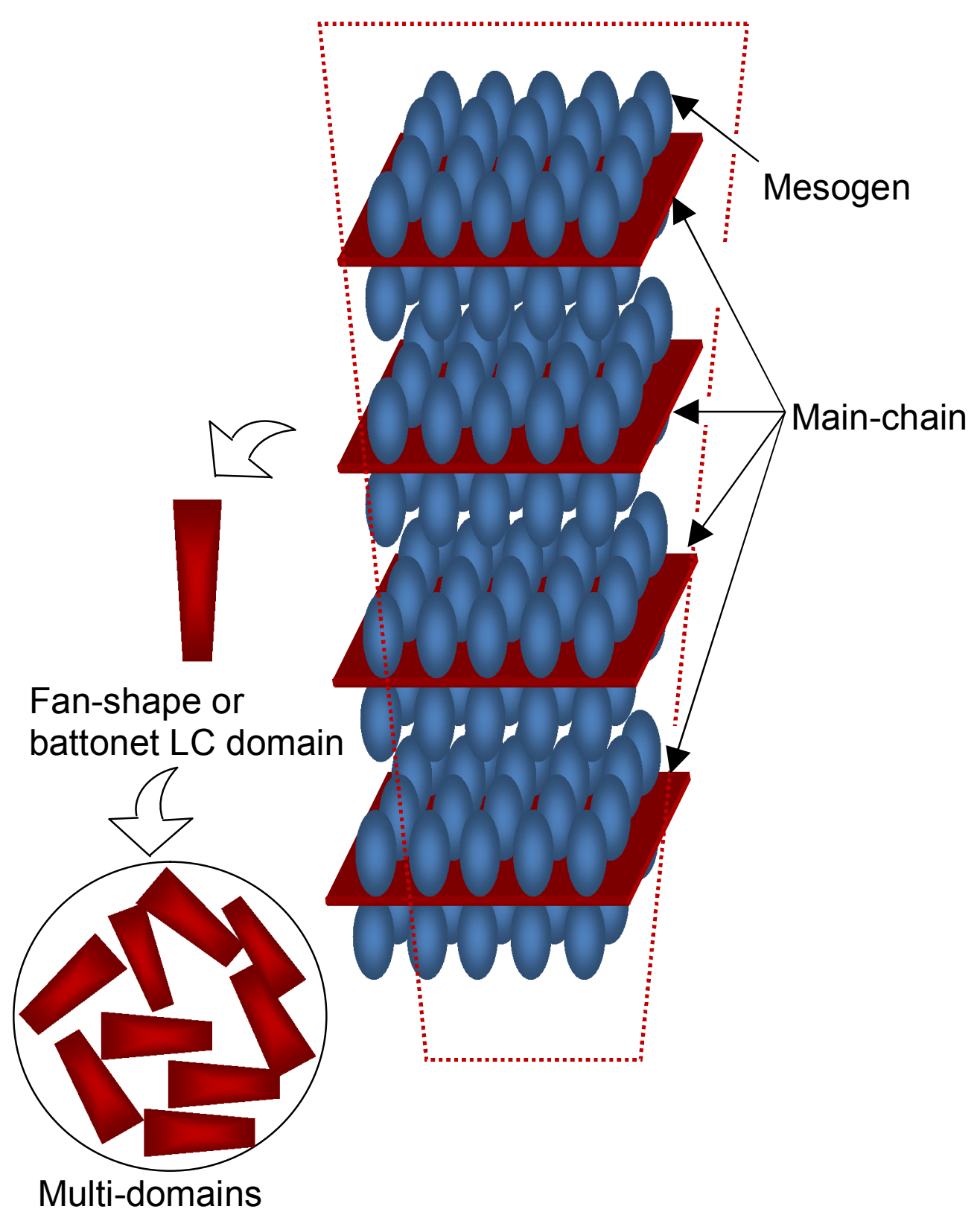

Fig. 6. Possible molecular aggregation scheme of SmA layer structure and formation of multi-domains.

Figure 7 shows the results of differential scanning calorimetry (DSC) measurements during heating and cooling. The two signals observed in each process indicate isotropic-SmA and SmA-glassy- state (solid) phase transitions. The transition temperatures are:.

glassy $12(12) \cdot \mathrm{SmA} \cdot 90(78) \cdot$ isotropic, in ${ }^{\circ} \mathrm{C}$ (bracket $=$ cooling process $)$. 


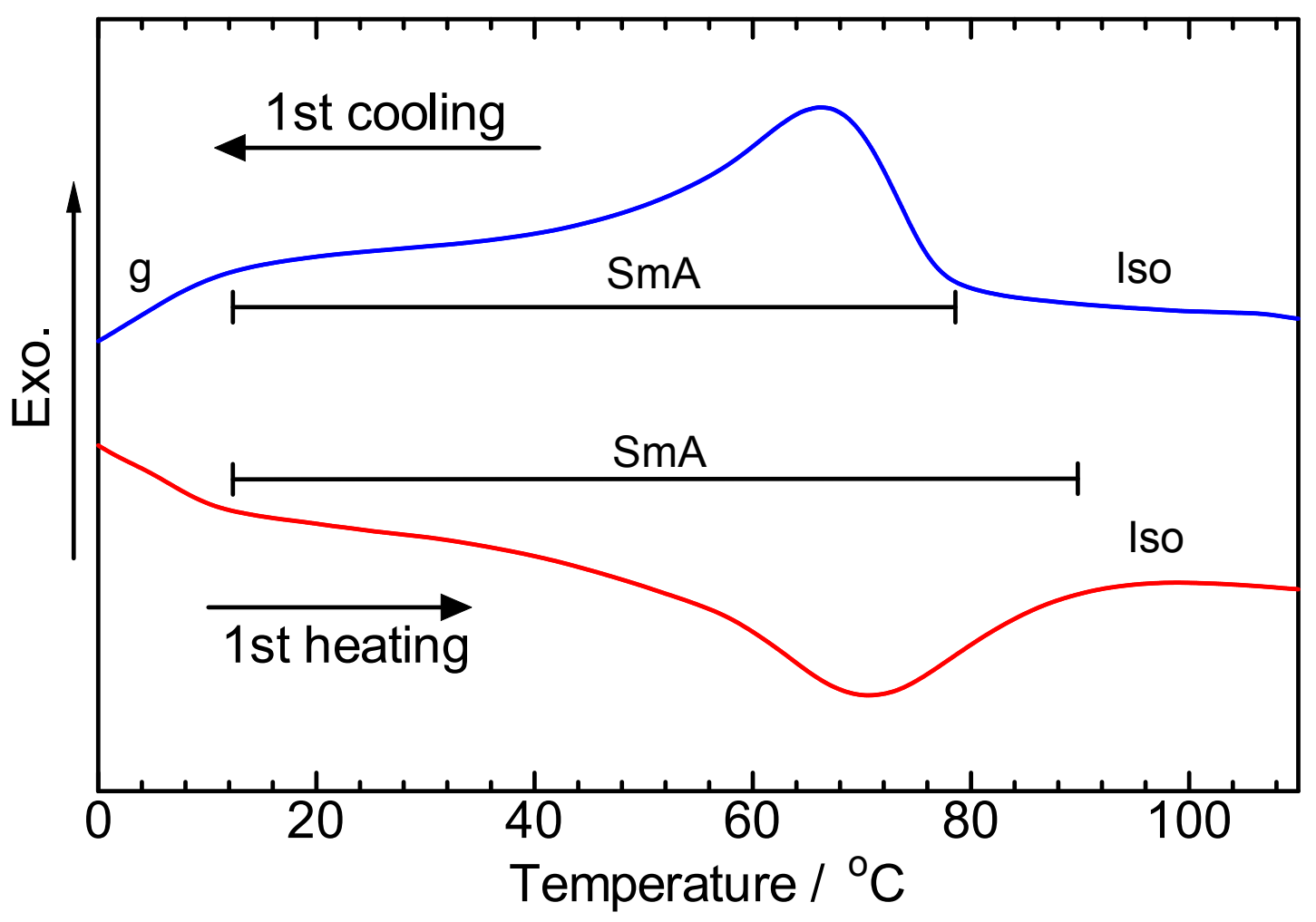

Fig. 7. DSC curves of polyITN-LC. $\mathrm{g}=$ glassy state, $\mathrm{SmA}=$ smectic A phase, Iso $=$ isotropic phase. Scan rate $=10^{\circ} \mathrm{C} / \mathrm{min}$

The polymer is soluble in tetrahydrofuran (THF), chloroform, dichloromethane, and toluene. We found that the films cast from these solutions showed fan-shaped texture via lyotropic liquid crystallinity (liquid crystallinity in solution), as shown in Figure 8 (cast from toluene). The sky-blue color of the thin sample is due to retardation color under crossed Nicols. In this case, the retardation color instead of original color of the polymer suggesting that the polymer film cast from the solutions exhibited liquid crystallinity in the evaporation process. As the solvent evaporated, an appropriate concentration range in the evaporation process afforded the formation of the liquid crystal aggregated form. Further solvent evaporation from the lyotropic LC afforded solid films with LC order. A diamond structure can be observed in the optical texture, as shown in Figure 8 (inset). This result indicates that the lyotropic LC phase of polyITN-LC is SmA, because the diamond structure and fan-shaped texture without stripes corroborate the presence of SmA. The bulk state of the polyITN-LC is in the form of SmA, and SmA film can be easily prepared by the solution process.

These results demonstrate that the polymer has amphotropic liquid crystallinity (both thermotropic and lyotropic liquid crystallinity). We could not confirm liquid 
Synthesis and Properties of a Low-Bandgap Liquid Crystalline $\pi$-Conjugated Polymer Hiromasa Goto, * Aohan Wang, Kohsuke Kawabata, Fan Yang

Journal of Materials Science, 48(21), 7523-7532, (2013)

crystallinity of polyITN-P because insoluble and infusible character.

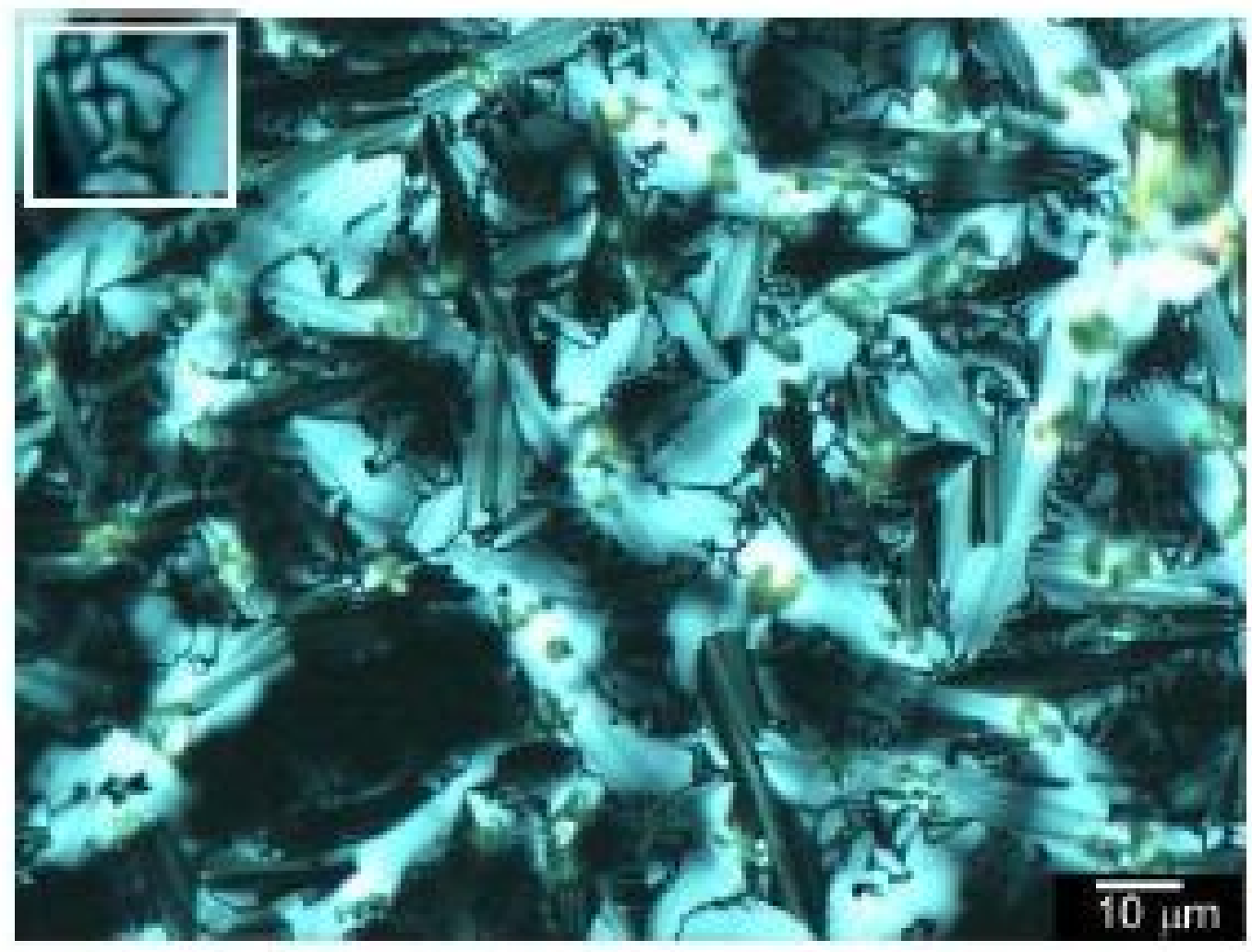

Fig. 8. Polarizing optical microscopy image of ITN-LC film cast from toluene solution.

\subsection{Dissipative liquid crystal texture}

One drop of polymer solution in toluene deposited onto a glass substrate produces a macroscopic fractal liquid crystal form, as shown in Figure 9(a). The concentric circles or concentric polygonal figures consist of liquid crystal multi-domains. The dissipative structure is formed by the convection processes taking place inside the drop deposited on the substrate. The periodic structure is spontaneously produced by a combined evaporative and convective process inside the drop of a polymer solution, such as Benard-Rayleigh convection [19]. Especially in this case molecular aggregation for formation of lyotropic liquid crystal is combined with fractal form to produce a liquid crystal organized structure. Figure $9(b, c)$ shows a magnified image of the ring texture showing periodic structure. Furthermore, this magnified image of the rings clearly shows that the lines consist of LC multi-domains. This appears similar to a Julio collective inside of a Mandelbrot set [20]. A possible formation mechanism of the LC fractal structure is shown in Figure 10. Figure 10(a,b) shows both the side view and top view, respectively, of an isotropic LC solution undergoing Benard-Rayleigh convection. 
Synthesis and Properties of a Low-Bandgap Liquid Crystalline $\pi$-Conjugated Polymer Hiromasa Goto,* Aohan Wang, Kohsuke Kawabata, Fan Yang Journal of Materials Science, 48(21), 7523-7532, (2013)

The convection produces concentric circles or polygonal figures. Evaporation via formation of lyotropic LC produces fractal concentric structure with LC order (Figure 10(d)). This process enables formation of a macroscopic LC dissipative structure.

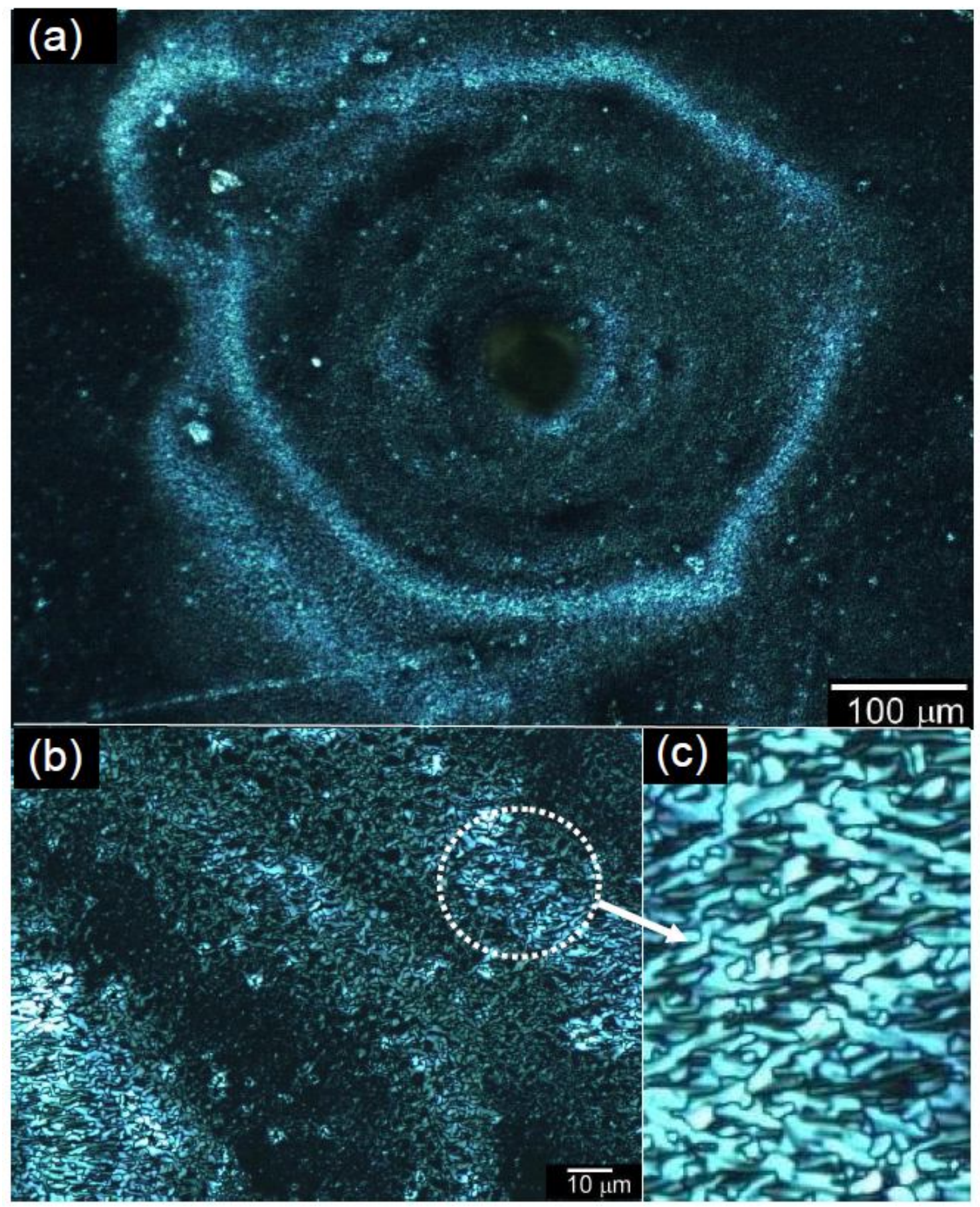

Fig. 9. Polarizing optical microscopy images of the polymer with lyotropic LC order prepared by the evaporation of one solution droplet (solvent $=$ toluene) deposited on glass substrate. (a) A dissipative structure. (b) A magnified image of the ring texture. (c) Liquid crystal multi-domains. 
Synthesis and Properties of a Low-Bandgap Liquid Crystalline $\pi$-Conjugated Polymer Hiromasa Goto,* Aohan Wang, Kohsuke Kawabata, Fan Yang

Journal of Materials Science, 48(21), 7523-7532, (2013)

This is the first example of a fractal figure consisting of LC domains. This bottom-up technique is combined with natural convection and aggregation to form a lyotropic LC. The liquid crystal structure is maintained and fixed after completion of the evaporation process. The polymer solid is stabilized in the form of the smectic liquid crystal.

(a)

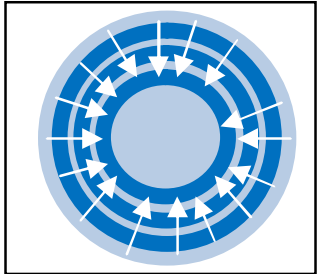

Top view

(b)

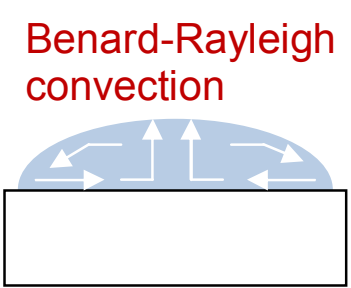

Side view

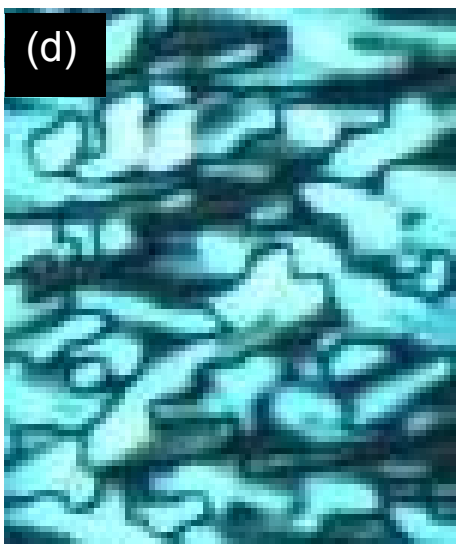

(c)
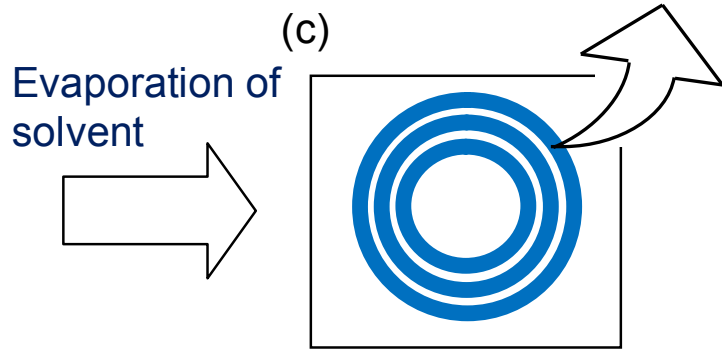

Completion of evaporation

Fig. 10. Possible formation process of dissipative structure of a macroscopic liquid crystal obtained via solvent evaporation and lyotropic liquid crystal (LC) formation. Top view (a) and side view (b) of isotropic LC solution undergoing Benard-Rayleigh convection. Completion of evaporation via formation of lyotropic LC produces concentric circles or concentric polygonal structure with LC order (c). The circles consist of LC domain structure (d). This process enables the formation of macroscopic LC fractal structure.

\subsection{Optical properties}

Figure 11(a) shows UV-vis absorption spectra of a chloroform solution and cast films. The band-edge bandgap of the polymer in solution was $2.0 \mathrm{eV}$ estimated from the onset of absorption $(600 \mathrm{~nm})$. The films with lyotropic LC order prepared by casting from chloroform solution and thermotropic LC order made by heat treatment both show a red shift compared to the polymer in the solution because co-planarity of the main chains is 
Synthesis and Properties of a Low-Bandgap Liquid Crystalline $\pi$-Conjugated Polymer Hiromasa Goto, ${ }^{*}$ Aohan Wang, Kohsuke Kawabata, Fan Yang Journal of Materials Science, 48(21), 7523-7532, (2013)

improved. Band-edge bandgap of the polymer films obtained from the onset of the absorption spectra was $1.7 \mathrm{eV}(698 \mathrm{~nm})$. The photoluminescence (PL) spectra of the polyITN-LC obtained in chloroform solution are shown in Figure 10(b). The emission from the polymer is observed at $509 \mathrm{~nm}$. The PL spectrum of the polymer film cast from a THF solution shows a weak peak at $490 \mathrm{~nm}$ (Figure 11(b)). The decrease in intensity may be due to quenching of the PL in the form of cast film. Interchain interaction of $\pi$-conjugated main chain often results in decrease of quantum yield. Although polyITN-LC may show photo-quenching in the form of LC as an aggregation state, emission from battonets of the polyITN-LC was observed (Figure 11(c)). This result demonstrates that polyITN-LC shows emission in the SmA LC order.

\subsection{Redox properties}

Figure 12(a) displays cyclic voltammetry (CV) results for the polyITN-LC solid film prepared on an indium tin oxide (ITO) - coated transparent glass electrode in $0.1 \mathrm{M}$ tetrabutyl ammonium perchlorate (TBAP)/acetonitrile ( $\mathrm{ACN}$ ) solution at a scanning rate of $10 \mathrm{mV} / \mathrm{s}$. A clear oxidation peak for the polymer is observed at $-0.24 \mathrm{~V}$. This process can be ascribed to generation of radical cations (polarons) as conducting carriers related to the electrochemical doping processes in the polymer. The perchlorate ion $\left(\mathrm{ClO}_{4}{ }^{-}\right)$from TBAP electrochemically doped the polymer surface with a SmA LC order. The low oxidation potential is derived from low-ionization potential of the low-bandgap skeleton based on the ITN. A reduction trough is observed in the CV at $-0.51 \mathrm{~V}$, which implies electrochemical dedoping. The CV result confirms that the present polymer is low-bandgap, and clearly shows electroactive doping-dedoping behavior.

Figure 12(b) indicates CIE color space of the polymer in the oxidized (doped) and reduced (dedoped) states confirming the change in color by the electrochemical potential applications. PolyITN-LC shows red and pale sky-blue colors in oxidized (full-doped, 1V) and reduced (dedoped) forms, respectively. Luminance parameter (Y) value was increased by the oxidation (Figure 12(b), right). The UV-Vis-NIR spectra of the polyITN-LC are shown in Figure 12(c). The absorption band due to $\pi-\pi^{*}$ transition of the main chain at $416 \mathrm{~nm}$ in the reduced state of the PITN-LC decreases, and a broad absorption band due to the doping appears at $>500 \mathrm{~nm}$ in the oxidized state. The results of the CIE color space and the absorption spectroscopy measurements indicate that polyITN-LC is electroactive, and shows electrochromism. This set of experimental findings demonstrates that the synthesis of the LC-ordered $\pi$-conjugated polymer was achieved. This system may serve as a first example of opto-electroactive conjugated polymer possessing a liquid crystal structure. 
Synthesis and Properties of a Low-Bandgap Liquid Crystalline $\pi$-Conjugated Polymer Hiromasa Goto,* Aohan Wang, Kohsuke Kawabata, Fan Yang Journal of Materials Science, 48(21), 7523-7532, (2013)

(a)

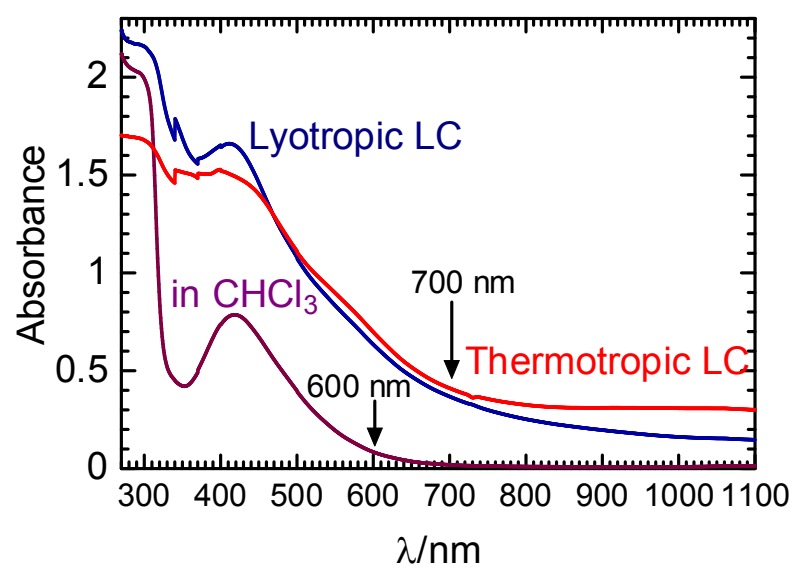

(b)
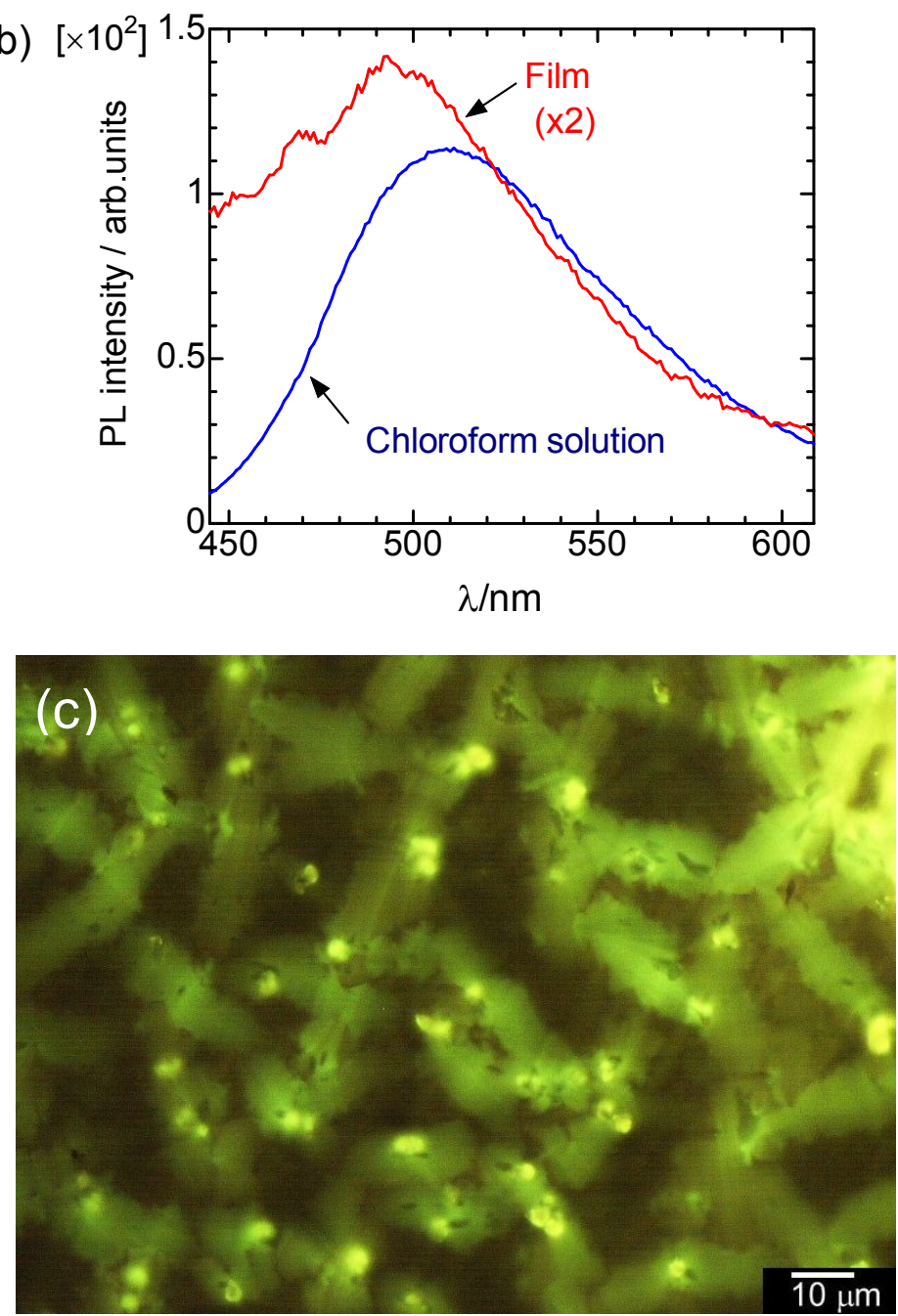

Fig. 11. (a) Optical absorption spectra of the polymer in chloroform solution, in thermotropic liquid crystal order (film), and lyotropic liquid crystal order (film). (b) Photoluminescence spectra of polyITN-LC in chloroform solution and cast film. (c) Photoluminescence optical microscopy image of polyITN-LC with lyotropic SmA LC 
Synthesis and Properties of a Low-Bandgap Liquid Crystalline $\pi$-Conjugated Polymer Hiromasa Goto, ${ }^{*}$ Aohan Wang, Kohsuke Kawabata, Fan Yang Journal of Materials Science, 48(21), 7523-7532, (2013)

(a) $\left[\times 10^{-5}\right]$

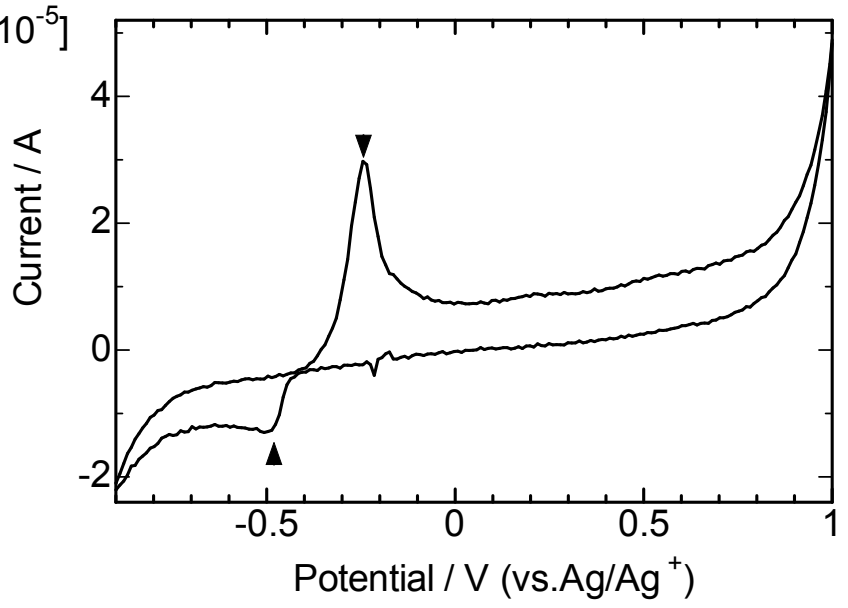

(b)

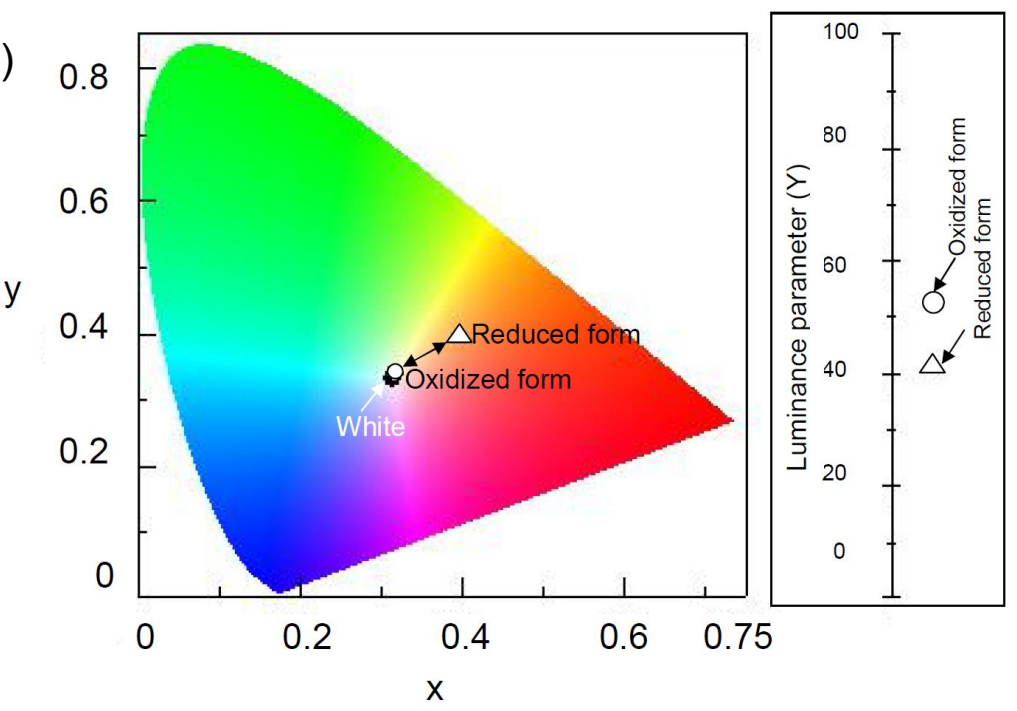

(c)

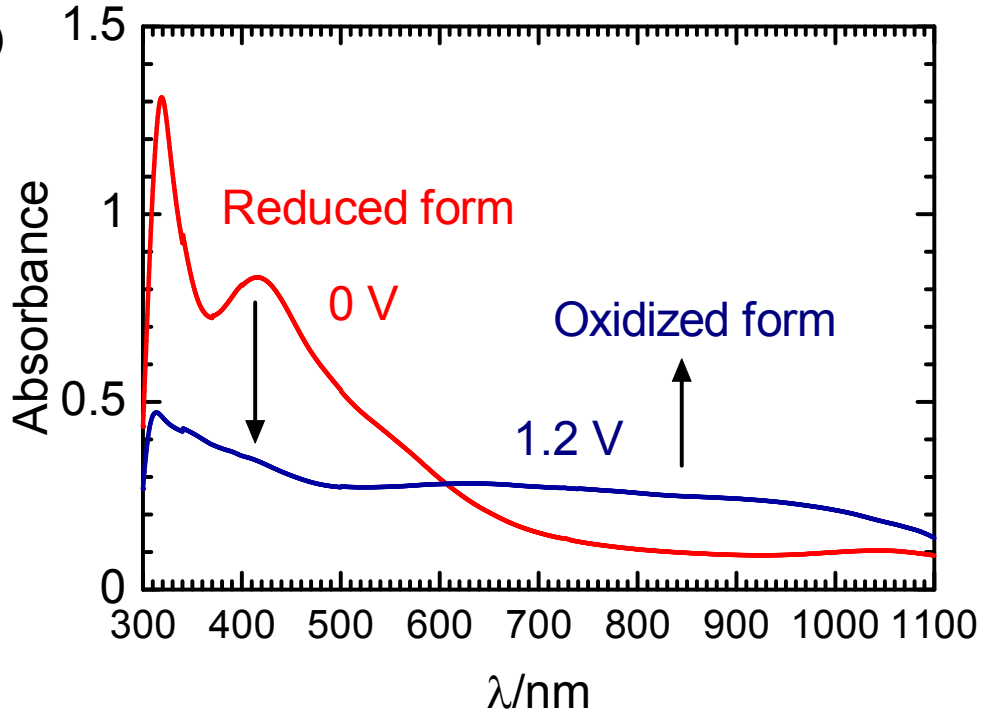


Fig. 12. (a): Cyclic voltammetry of polyITN-LC vs. $\mathrm{Ag} / \mathrm{Ag}^{+}$in $0.1 \mathrm{M}$ tetrabutyl ammonium perchlorate/acetonitrile solution. (b): CIE color space of polyITN-LC in electrochemically oxidized and reduced form. (b, right): Luminance parameter (Y). (c): UV-Vis-NIR absorption spectra of polyITN-LC in oxidized and reduced form.

\section{Conclusions}

We synthesized an isothianaphthene-based liquid crystal conjugated polymer. The polymer shows both thermotropic and lyotropic liquid crystallinities. The formation of macroscopic LC fractal structure was discussed. Liquid crystal emission of the polyITN-LC was observed. Through this research preparation of an opto-electroactive liquid crystalline conjugated polymer has been achieved. This new approach may provide an avenue to create novel optically functional electro-plastics such as liquid crystal emission devices and liquid crystal photovoltaics.

\section{Acknowledgments}

We would like to thank the Chemical Analysis Center and the Glass Work Shop of the University of Tsukuba. We wish to express our sincere appreciation to Dr. R. H. L. Kiebooms (European Patent Office) for encouragement and advice. Pyrimidine LC precursors were generously provided by Midori Chemical (Midori Kagaku, Japan).

\section{References}

[1]Bundgaard E, Krebs F (2007) Solar Energy Mater Solar Cells 91:954

[2]Jung Y, Kim H, Park J, Lee J, Lee S, Lee Y, Shim H (2008) Polym Chem 45:3573

[3] Hou J, Chen H, Zhang S, Li G, Yang Y (2008) J Am Chem Soc 130:16144.

[4]Chen S, Lee C (1996) Polymer 37: 519

[5] Wudl F, Kobayashi M, Heeger A (1984) J Org Chem 49:3382

[6] Li P, Fenwick O, Yilmaz S, Breusov D, Caruana D, Allard S, Scherf U, Cacialli F (2011) Chem Comm 47:8820

[7] Kobayashi M, Colaneri N, Boysel M, Wudl F, and Heeger A (1985) J Chem Phys $82: 5715$

[8] He Y, Chen C, Richard E, Dou L, Wu Y, Li G, Yang Y (2012) J Mater Chem 22:13391

[9] Hsieh C, Cheng Y, Li P, Chen C, Dubosc M, Liang R, Hsu C (2010) J Am Chem Soc $132: 4887$

[10] Price S, Stuart A, You W (2010) Macromolecules 43:797

[11] Zhu Z, Swager T (2002) J Am Chem Soc 124:9670 
Synthesis and Properties of a Low-Bandgap Liquid Crystalline $\pi$-Conjugated Polymer Hiromasa Goto, * Aohan Wang, Kohsuke Kawabata, Fan Yang

Journal of Materials Science, 48(21), 7523-7532, (2013)

[12] Zhang Z, Tang H, Liang X, Shi G (2006) J Electroanal Chem 587:308

[13] Innami Y, Kawashima H, Kiebooms R, Aizawa H, Matsuishi K, Goto H (2012) Materials 5:317

[14] Wang A, Kawabata K, Goto H (2013) Materials 6:2218

[15] Yang Q, Kim J, Frisbie C, Hillmyer M (2008) Macromolecules 41:5563

[16] Kawabata K, Goto H (2012) J Mater Chem 22: 23514

[17] Chen W, Bowmaker G, Seakins J, Cooney R (2002) Appl Spect 56:909

[18] Neugebauer H, Kvarnström C, Brabec C, Sariciftci N, Kiebooms R, Wudl F, Luzzati S (1999) J Chem Phys 110: 12108

[19] Yunker P, Still T, Lohr M, Yodh A (2011) Nature 476:308

[20] Karthaus O, Maruyama N, Cieren X, Shimomura M, Hasegawa H, Hashimoto T (2000) Langmuir 16:6071 